The University of Southern Mississippi

The Aquila Digital Community

Faculty Publications

$2-1-2003$

\title{
Efficient Incorporation of CoA, NAD and FAD Into RNA by In Vitro Transcription
}

Faqing Huang

University of Southern Mississippi, Faqing.Huang@usm.edu

Follow this and additional works at: https://aquila.usm.edu/fac_pubs

Part of the Chemistry Commons

\section{Recommended Citation}

Huang, F. (2003). Efficient Incorporation of CoA, NAD and FAD Into RNA by In Vitro Transcription. Nucleic Acids Research, 31(3), 1-8.

Available at: https://aquila.usm.edu/fac_pubs/9216

This Article is brought to you for free and open access by The Aquila Digital Community. It has been accepted for inclusion in Faculty Publications by an authorized administrator of The Aquila Digital Community. For more information, please contact Joshua.Cromwell@usm.edu. 


\title{
Efficient incorporation of CoA, NAD and FAD into RNA by in vitro transcription
}

\author{
Faqing Huang* \\ Department of Chemistry and Biochemistry, University of Southern Mississippi, Hattiesburg, MS 39406-5043, USA
}

Received June 24, 2002; Revised August 26, 2002; Accepted November 25, 2002

\begin{abstract}
Protein enzymes frequently recruit small molecule coenzymes to perform a variety of biochemical reactions. While the catalytic activities of RNA have been expanding rapidly, a similar strategy for RNA to utilize coenzymes and to increase its functional capabilities has yet to be demonstrated. A general in vitro transcription procedure has been developed to efficiently prepare RNA with coenzymes CoA, NAD and FAD covalently attached to the $5^{\prime}$ end. These adenosine-containing coenzymes initiate transcription under the $\mathrm{T7}$ class II promoter by T7 RNA polymerase. In addition to the three coenzymes, other adenosine-containing molecules may be incorporated into the first nucleotide position of RNA as well. This method provides easy access to CoA-, NAD- and FAD-RNA, which may find broad applications in generating coenzymeutilizing ribozymes. In addition, both oxidized FAD and reduced NADH are highly fluorescent. NADH-RNA and FAD-RNA can therefore be used as probes for DNA/RNA detection and for structural investigation of RNA function by fluorescence spectroscopy.
\end{abstract}

\section{INTRODUCTION}

Coenzymes are a group of small organic molecules with distinct chemical functionalities, which are usually unavailable or insufficient in the side chains of the 20 naturally occurring amino acids. A number of metabolic protein enzymes require coenzymes to act as accessory molecules to provide necessary reactivities. Three common coenzymes, coenzyme A (CoA), nicotinamide adenine dinucleotide (NAD) and flavin adenine dinucleotide (FAD), carry out a variety of acyl group transfer and electron/hydride transfer reactions in metabolism. Although CoA, NAD and FAD function in very different chemical capacities, they share some surprisingly common structural features: a ribonucleoside adenosine at one end, a chemically functional group (pantetheine, nicotinamide or riboflavin) at the other and a pyrophosphate connecting the two groups.

Because the structures and functions of these coenzymes are conserved in all kingdoms, CoA, NAD and FAD are believed to have existed since the last common ancestor of life on Earth (1). Furthermore, the fact that all these coenzymes contain an adenosine group, one of the four essential building monomers for RNA, suggests the plausibility of their existence and biological function in a more ancient world, as first hypothesized by White (2,3). Recently, we demonstrated RNA-catalyzed synthesis of these three coenzymes from their corresponding precursors, phosphopantetheine, nicotinamide mononucleotide (NMN) and flavine mononucleotide (FMN) (4), providing strong experimental evidence of possible coenzyme synthesis and utilization in the RNA world (5).

Current known ribozymes, either naturally occurring or isolated in vitro, however, do not utilize these coenzymes to perform chemistries as protein enzymes do. A recent report has demonstrated RNA-catalyzed acyl-CoA syntheis (6), and we have independently isolated ribozymes capable of catalyzing thioester formation (7). Yet, RNA catalytic activities involving NAD and FAD redox chemistry have not been shown to be within the functional capacity of RNA. Because of the plausible prominent roles of coenzymes in an early RNA world and the deficiency in RNA component diversity, we believe that RNA should be able to recruit and utilize coenzymes in a broad range of biochemical reactions. Since the development of in vitro selection (SELEX) (8-10), numerous artificial ribozymes have been isolated from random RNA libraries (11). New RNA sequences capable of catalyzing acyl group transfer and redox reactions by using CoA, NAD and FAD are expected to join the ever-growing list of artificial ribozymes.

Covalent coupling of these coenzymes to RNA sequences not only eliminates the requirement of coenzyme binding by ribozymes, but may also provide a practical means of selection based on the coenzyme reactivity. We report here the development of a general method of incorporating CoA, NAD and FAD into RNA through in vitro transcription. This procedure may be used to construct coenzyme-linked RNA libraries for in vitro selection or to prepare coenzyme-coupled specific RNA sequences for other purposes, such as fluorescent labeling and detection of RNA/DNA, investigation of RNA structure and studying RNA-RNA interaction and RNA-protein interaction.

\section{MATERIALS AND METHODS}

\section{Reagents}

3'-Dephospho-coenzyme A (De-P-CoA), NAD and FAD were purchased from Sigma-Aldrich (St Louis, MO) (catalog nos

*Tel: +1 601266 4371; Fax: +1 601266 6075; Email: faqing.h.huang@usm.edu 
D3385, N6522 and F6625 for De-P-CoA, NAD and FAD, respectively). ATP, UTP, GTP and CTP were from Boehringer Mannheim (Indianapolis, IN). T7 RNA polymerase came from Epicentre Technologies (Madison, WI). RNase inhibitor, Taq DNA polymerase, nuclease P1, DTT, dATP, dTTP, dGTP and dCTP were obtained from Promega Life Science Technologies (Madison, WI). Thiopropyl Sepharose 6B was from Amersham Pharmacia Biotech (Piscataway, NJ). DNA oligonucleotides were from either Operon Technologies (Alameda, CA) or Integrated DNA Technologies (Coralville, IA).

\section{Transcription, DNazyme cleavage and gel electrophoresis}

Transcription promoter sequence was derived from the $\mathrm{T} 7$ class II promoter ( $\phi 2.5)$ (Fig. 1) (12). The RNA sequence was from a previously isolated coenzyme-synthesizing ribozyme (4), CoES7, with the sequence AGGGAAGTGCTACCACAACUUUAGCCAUAAUGUCACUUCUGCCGCGGGCAUGCGGCCAGCCA. Transcription was carried out under the following standard conditions: $1 \mathrm{mM}$ each of ATP, UTP, GTP and CTP, $40 \mathrm{mM}$ Tris (pH 8.0), $6 \mathrm{mM} \mathrm{MgCl}_{2}, 2 \mathrm{mM}$ spermidine, $0.01 \%$ Triton X-100, $5 \mathrm{mM}$ DTT, $0.2 \mathrm{U} / \mu 1$ RNase inhibitor, $0.2 \mu \mathrm{M}$ DNA templates and $5 \mathrm{U} / \mu \mathrm{l}$ T7 RNA polymerase. In addition, different concentrations of coenzymes, De-P-CoA, NAD and FAD, were included to initiate transcription. For quantitation purposes, $\left[\alpha-{ }^{32} \mathrm{P}\right] \mathrm{ATP}$ (NEN Life Science, Boston, MA) was also added to transcription solutions to internally label RNA transcripts. All transcription reactions were allowed to proceed for $2 \mathrm{~h}$ at $37^{\circ} \mathrm{C}$ before product analysis by $8 \%$ denaturing PAGE.

To physically separate CoA-RNA from pppRNA by PAGE, a different RNA sequence of $35 \mathrm{nt}$ was designed to allow a specific DNazyme (13) cleavage after transcription. Two DNA oligos with the sequences CAGTAATACGACTCACTATTAGGGAAGCGGGCATGCGGCCAGCCATAGCCGATCA and TGATCGGCTATGGCTGGCCGCATGCCCG were used to construct (by PCR) a DNA duplex template, from which a 35 nt RNA sequence AGGGAAGCGGGCAUGCGGCCAGCCAUAGCCGAUCA was transcribed. A DNazyme (with the sequence TGATCGGCTAGGCTAGCTACAACGAGGCTGGCCGC) was then used to specifically cleave off a portion of the $3^{\prime}$ heterogeneous ends of both CoA-RNA and pppRNA, yielding a homogeneous length of $25 \mathrm{nt} \mathrm{CoA-RNA}$ and pppRNA (Fig. 3A). Similar transcription conditions were used with the exception of different concentrations of ATP (indicated in Fig. 3B). Transcribed RNA $(\sim 2 \mu \mathrm{M})$ was incubated with $0.4 \mu \mathrm{M}$ DNazyme for $60 \mathrm{~min}$ at $37^{\circ} \mathrm{C}$ in $50 \mathrm{mM} \mathrm{MgCl} 2$ and $50 \mathrm{mM}$ Tris, $\mathrm{pH}$ 8.0. RNA was then analyzed by $12 \%$ denaturing PAGE.

\section{RNA quantitation}

After PAGE, individual RNA bands were visualized through phosphorimaging (Molecular Imager; Bio-Rad Laboratories, Hercules, CA). Quantitation of different RNA bands was achieved through profile integration after horizontal background subtraction. Coenzyme-RNA (CoE-RNA) yields were calculated based on relative intensity of pppRNA bands $(N$, $N+1$ and $N+2$ bands) in the absence of coenzymes (Fig. 2A, lane 1). To confirm the validity of the calculation, DNazymecleaved pppRNA and CoA-RNA were quantitated directly

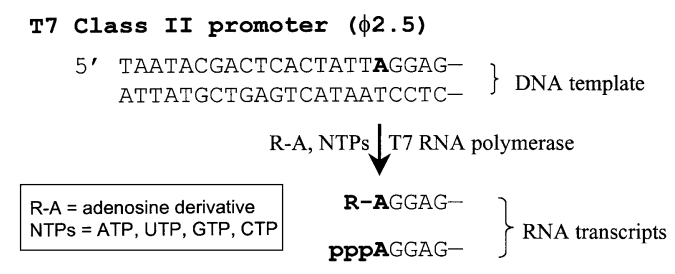

Figure 1. Adenosine derivative-initiated transcription under the T7 class II promoter ( $\phi 2.5)$ (12). In addition to nucleoside 5'-triphosphates (NTPs), an adenosine derivative (R-A) is added to the transcription solution to serve as the transcription initiator to produce R-ARNA. ATP competes with R-A for transcription initiation, resulting in normal RNA with $5^{\prime}$ triphosphate, pppRNA.

after gel resolution of CoA-RNA from pppRNA. In addition, CoA-RNA yield was determined independently by thiopropyl Sepharose 6B affinity chromatography based on its free sulfhydryl group.

\section{Fluorescence emission spectra of FAD-RNA}

FAD-RNA was prepared by transcription under the standard conditions in the presence of $4 \mathrm{mM} \mathrm{FAD} \mathrm{(no}\left[\alpha-{ }^{32} \mathrm{P}\right] \mathrm{ATP}$ was added). Two consecutive purification procedures were used to ensure that purified FAD-RNA does not contain free FAD from the transcription solution. After $4 \mathrm{~h}$ of transcription at $37^{\circ} \mathrm{C}$, the RNA sample was loaded onto a Microcon filter (M30; Millipore Corp., Bedford, MA) and centrifuged at $14000 \mathrm{~g}$ to a final volume of $\sim 5 \mu \mathrm{l}$. Then, $200 \mu \mathrm{l}$ of water was added to the Microcon filter and centrifuged down to $\sim 5 \mu \mathrm{l}$ solution left on the filter. An equal volume of gel loading solution containing $7 \mathrm{M}$ urea and $0.02 \%$ xylene cyanol was added to the filter. Recovered RNA from the filter was heated at $90^{\circ} \mathrm{C}$ for $1 \mathrm{~min}$ and loaded onto an $8 \%$ denaturing polyacrylamide gel. After gel electrophoresis, RNA bands (containing both pppRNA and FAD-RNA) were located by UV shadowing and excised from the gel. RNA was then eluted and recovered by ethanol precipitation.

Fluorescence emission spectra of $0.1 \mu \mathrm{M}$ free FAD and $0.1 \mu \mathrm{M}$ FAD-RNA (after FAD-RNA yield correction according to Fig. 2D) were recorded with an ISS PC1 fluorometer (Champaign, IL). FAD has two absorption peaks, located at $\sim 370$ and $450 \mathrm{~nm}$ (with $\varepsilon_{450}>\varepsilon_{370}$ ), respectively. Its fluorescence emission spectrum peaks at $\sim 530 \mathrm{~nm}$. When FAD is excited at $450 \mathrm{~nm}$, the Raman scatter peak co-localizes with the fluorescence emission peak at $\sim 530 \mathrm{~nm}$. To avoid the interference of the Raman scatter with fluorescence emission, both free FAD and FAD-RNA were excited at $370 \mathrm{~nm}$. To see the effect of RNA on FAD fluorescence emission intensity, FAD-RNA emission spectra were also recorded before and after complete digestion by nuclease P1. Digestion of RNA was achieved by adding $1 \mathrm{U}$ nuclease $\mathrm{P} 1$ in a buffer containing $10 \mathrm{mM} \mathrm{NaAc}\left(\mathrm{pH} \mathrm{5.2)}\right.$ and $0.4 \mathrm{mM} \mathrm{ZnCl}_{2}$. The effect of urea on FAD-RNA fluorescence was also investigated with two different concentrations of urea, 4 and $7 \mathrm{M}$.

\section{RESULTS}

\section{Adenosine derivative-initiated transcription}

We have previously demonstrated ATP-initiated transcription (4) under the T7 class II promoter (12). As an extension of the 
work, we sought to develop a general transcription method for preparation of RNA with adenosine derivatives linked to its $5^{\prime}$ end. Because many biological cofactors, such as coenzymes CoA, NAD and FAD, contain an adenosine group, they may be used as the transcription initiators under the T7 class II promoter, leading to the synthesis of cofactor-linked RNA, as shown in Figure 1. Because CoA contains a $3^{\prime}$ phosphate group that can block $3^{\prime}$ extension, dephosphorylated CoA (De-P-CoA) must be used to initiate transcription.

Transcription of CoES7 RNA was carried out under the standard conditions, with the addition of various concentrations of coenzymes. Figure $2 \mathrm{~A}$ shows the reaction scheme (top) and results from transcription in the absence or presence of $4 \mathrm{mM}$ coenzymes (bottom). After fractionation by PAGE, different RNA bands became clearly visible. In the absence of any coenzymes (lane 1), transcription produced three pppRNA bands in decreasing order: the full-length RNA transcripts ( $N$ band) and N+1 and N+2 bands (with one and two extra nucleotides added at the $3^{\prime}$ end of full-length RNA). Their relative yields were 59,35 and 6\% (from bottom to top), respectively. When one of the three coenzymes, De-P-CoA, NAD or FAD, was added to the transcription solution (lanes 2-4), no additional RNA bands were generated. However, the relative intensity of the three RNA bands in each lane changed considerably when compared with that of lane 1 . In all three lanes (lanes 2-4), the intensity of the $N$ band RNA decreased, while both the N+1 and N+2 bands were significantly higher than those in lane 1 . In the presence of $4 \mathrm{mM}$ De-P-CoA (lane 2 ), the relative intensity of the three bands changed to 40,45 and $15 \%$ (from bottom to top). The relative RNA band intensities were 30, 49 and 21\% for both NAD- and FADinitiated transcription (lanes 3 and 4). These changes in RNA band relative intensity resulted from the incorporation of coenzymes (De-P-CoA, NAD and FAD) into RNA. When one of these coenzymes is incorporated at the $5^{\prime}$ end of RNA, the coenzyme-linked RNA (CoE-RNA) behaves as if an extra normal nucleotide is added to the pppRNA. Therefore, the $N$ band CoE-RNA co-migrates with the $N+1$ band pppRNA, while the $N+1$ band CoE-RNA and the $N+2$ band pppRNA run together in the gel. The $N+2$ band CoE-RNA was barely visible because of its low intensity. To confirm the incorporation of coenzymes into RNA under the class II promoter, three independent experiments were performed: resolution of CoA-RNA from pppRNA by gel electrophoresis after

Figure 2. Incorporation of De-P-CoA, NAD and FAD into RNA under standard transcription conditions, with additional coenzymes as shown. The RNA sequence used for the experiments was that of a coenzymesynthesizing ribozyme, CoES7, previously isolated by in vitro selection (4). (A) Transcription of coenzyme-linked RNA in the presence of different coenzymes. (Top) Transcription reactions in the presence of coenzymes. (Bottom) Various transcription products in the absence (lane 1) and presence of $4 \mathrm{mM}$ coenzymes (lanes $2-4)$. The three normal RNA bands $(N$, $N+1$ and $N+2$ pppRNA, lane 1) and coenzyme-RNA bands $(N$ and $N+1$ CoE-RNA) are resolved by $8 \%$ denaturing PAGE. (B) Total RNA (pppRNA and CoA-RNA) yield and CoA-RNA yield at different 3'-dephospho-CoA (De-P-CoA) concentrations. Total RNA yields are relative to the RNA yield in the absence of De-P-CoA, while CoA-RNA yield represents the percentage of CoA-RNA over the total RNA at the indicated De-P-CoA concentration. (C) Relationships among total RNA yield, NAD-RNA yield and NAD concentration. (D) Effect of FAD concentration on total RNA yield and FAD-RNA yield.
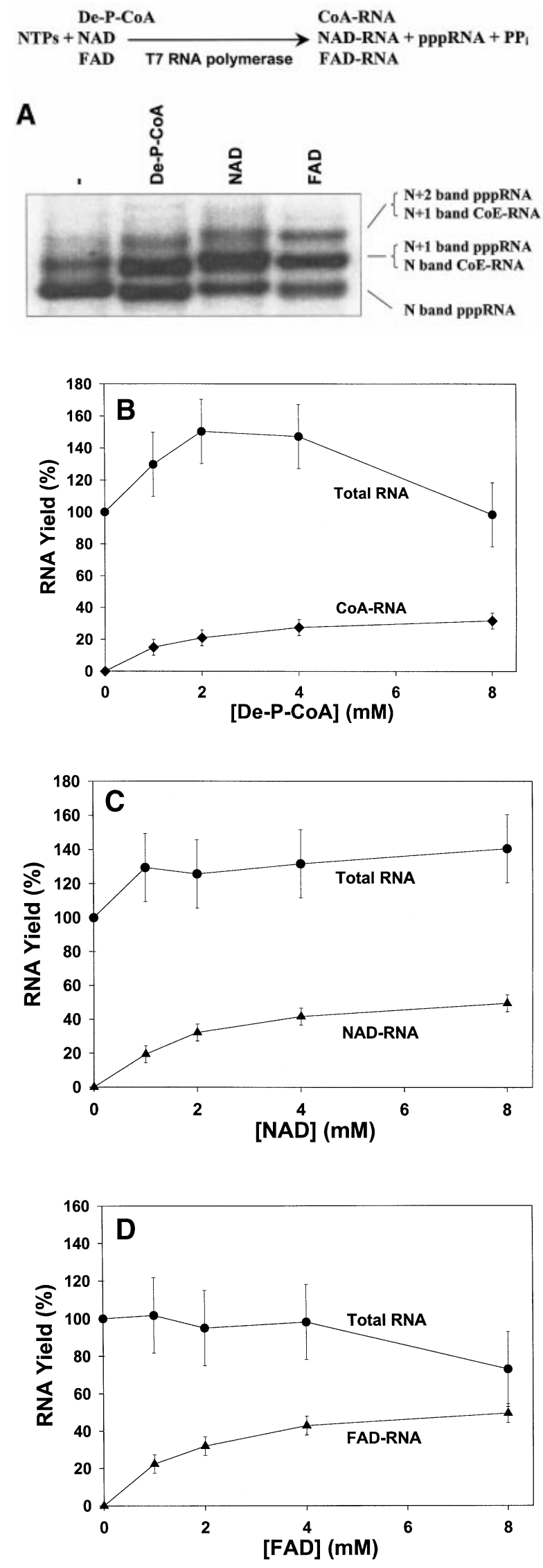
DNazyme cleavage, measurement of the free sulfhydryl group of CoA-RNA and measurement of the fluorescence emission spectrum of FAD-RNA (see below).

Both the total RNA yields and CoE-RNA yields depend on coenzyme concentrations, as shown in Figure 2B-D. Among the three coenzymes included in this investigation, individual coenzymes appear to have some different effects on transcription. For De-P-CoA incorporation (Fig. 2B), the total RNA yield (pppRNA + CoA-RNA) first increases slightly with the concentration of De-P-CoA, indicating that De-PCoA stimulates transcription to some extent at low concentrations. However, at higher concentrations, the stimulatory effect of De-P-CoA disappears, and eventually becomes inhibitory. On the other hand, the CoA-RNA yield increases when higher concentrations of De-P-CoA are added to the transcription solution. CoA-RNA reaches $30-35 \%$ of total RNA synthesized at 4-8 mM De-P-CoA. Both NAD and FAD have a similar effect on relative CoE-RNA yields (Fig. 2C and D). NAD-RNA and FAD-RNA first increase rapidly with the concentration of NAD and FAD, and then plateau at high NAD and FAD concentrations. In the presence of 4-8 mM NAD or FAD, transcription produces 35-40\% NAD-RNA or FAD-RNA over the total RNA synthesized. On the other hand, NAD and FAD appear to exert different effects on total RNA yields. While different concentrations of NAD slightly stimulate transcription (Fig. 2C), FAD appears to have a somewhat inhibitory effect, especially at high concentrations (>4 mM) (Fig. 2D). Results in Figures 2B-D were derived from two independent transcription experiments. From our other experiments using this transcription method to prepare CoE-RNA, we have found that the relative CoE-RNA yields in Figure 2B-D have been very reproducible. However, some degree of variation in total RNA yields may exist among different experiments. In the presence of $>8 \mathrm{mM}$ coenzymes (especially De-P-CoA and FAD), total RNA yields can be significantly lower than under the standard transcription conditions (data not shown).

To physically resolve CoE-RNA from pppRNA by gel electrophoresis, a DNazyme (13) was designed to trim off the $3^{\prime}$ heterogeneous ends of RNA (Fig. 3A). Although transcription produces $N, N+1$ and $N+2$ bands of both pppRNA and CoA-RNA, the DNazyme cuts at a specific site and yields both CoA-RNA and pppRNA of the same length of $25 \mathrm{nt}$. Comparing lanes 1 and 2 (Fig. 3B), the upper band in lane 2 represents CoA-RNA (33\%), while the bottom band is pppRNA (it is not clear why there was a weak band above the expected product bands, but it represented well below $10 \%$ of total RNA). The CoA-RNA yield agrees well (within the experimental error of 5\%) with the results from Figure 2B. When the concentration of De-P-CoA was maintained at $4 \mathrm{mM}$ and the ATP concentration was reduced from 1 to $0.5,0.2$ and $0.1 \mathrm{mM}$ (keeping GTP, CTP and UTP concentrations at 1 $\mathrm{mM}$ ), the relative CoA-RNA yield increased from 33 to 46,52 and $56 \%$. On the other hand, the total RNA yield decreased from $110 \%$ (relative to the RNA obtained at $1 \mathrm{mM}$ ATP and $0 \mathrm{mM}$ De-P-CoA) to 85,44 and $12 \%$. The seemingly more intense bands in lanes 3 and 4 relative to lane 2 resulted from higher ratios of $\left[\alpha_{-}{ }^{32} \mathrm{P}\right] \mathrm{ATP} / \mathrm{ATP}$ when the ATP concentration was lowered.

The effect of $3^{\prime}$ blocked adenosine derivatives on transcription was also investigated by gel electrophoresis after
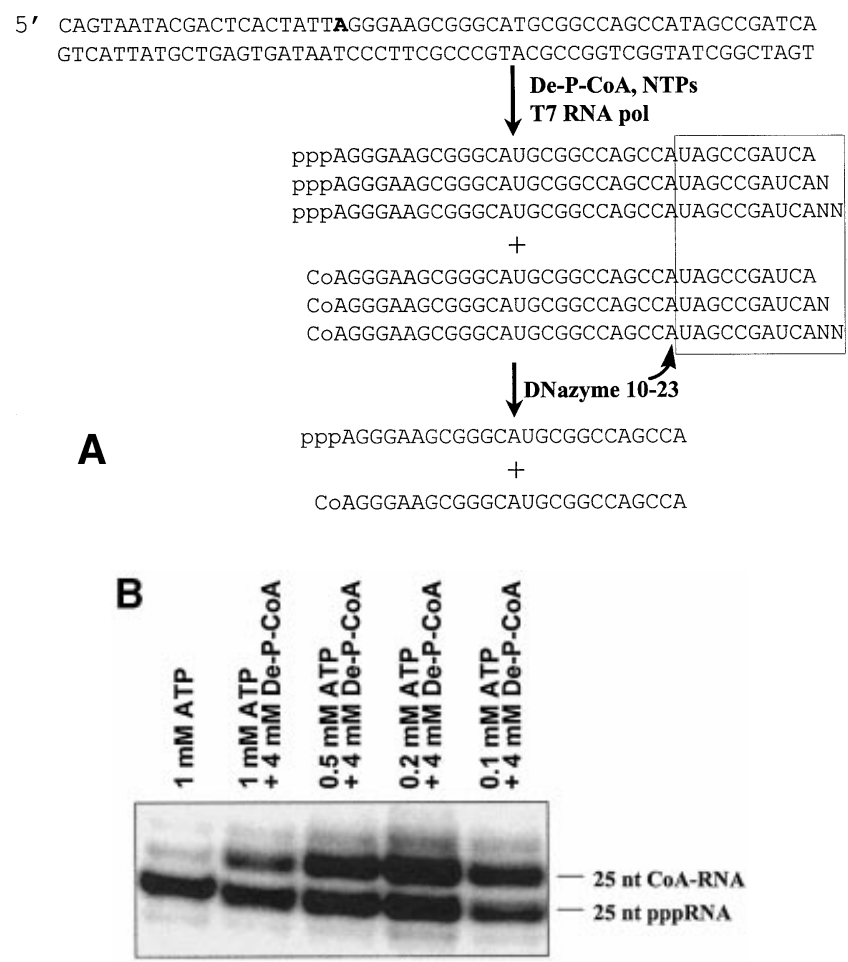

Figure 3. Separation of CoA-RNA and pppRNA by PAGE after a specific cleavage by a DNazyme (13). (A) Scheme of RNA from transcription and after DNazyme cleavage. In the presence of De-P-CoA, transcription produces a mixture of pppRNA $(N, N+1$ and $N+2$ bands, $N=35 \mathrm{mer})$ and CoA-RNA $(N, N+1$ and $N+2$ bands). A DNazyme (5'-TGATCGGCTAGGCTAGCTACAACGAGGCTGGCCGC) cuts at a specific location (marked by an arrow), resulting in both pppRNA and CoA-RNA with a defined length of $25 \mathrm{nt}$. The bold $\mathrm{A}$ is the initiating nucleotide of transcription. RNA portions removed by the DNazyme are boxed. (B) Effect of ATP and De-P-CoA on transcription. After transcription, RNA was digested by the DNazyme and then fractionated by $12 \%$ denaturing gel electrophoresis. All transcription reactions were performed with the same concentration of $\left[\alpha-{ }^{32} \mathrm{P}\right] \mathrm{ATP}(0.1 \mu \mathrm{M})$. The relative ratios of $\left[\alpha-{ }^{32} \mathrm{P}\right] \mathrm{ATP} /$ ATP from lane 1 to 5 were $1,1,2,5$, and 10 , respectively. These different ratios were considered in the calculation of total RNA yields (relative to lane 1).

DNazyme cleavage of RNA. At $4 \mathrm{mM}$, neither CoA nor 3'AMP had a detectable effect on either normal RNA transcription (with no coenzymes) or CoA-RNA transcription (in the presence of $4 \mathrm{mM}$ De-P-CoA) (results not shown). The result is in excellent accordance with the specific adenosine derivative-initiated transcription, which requires a free $3^{\prime}$ hydroxyl group for RNA extension.

To further confirm the yields of CoE-RNA derived from the PAGE and phosphorimaging analyses, the CoA-RNA yield was independently determined through its $5^{\prime}$ free sulfhydryl group by thiopropyl Sepharose 6B affinity chromatography. CoES7 RNA was prepared under $1 \mathrm{mM}$ ATP and $4 \mathrm{mM}$ De-P$\mathrm{CoA}$ and purified by membrane filtration with Microcon 30 . After passing the RNA through a thiopropyl Sepharose 6B column followed by thorough washing with water, 30-35\% of loaded RNA was retained. The retained RNA was completely released from the column by $30 \mathrm{~min}$ incubation with $20 \mathrm{mM}$ DTT in $20 \mathrm{mM}$ Tris, $\mathrm{pH}$ 8.5. The result not only confirms that CoA-RNA is synthesized by T7 RNA polymerase in the 
presence of De-P-CoA, but also corroborates quantitatively the results from the PAGE and phosphorimaging analyses. Further confirmation of CoE-RNA synthesis comes from the following fluorescence measurement of FAD-RNA.

\section{Fluorescence emission spectra}

Fluorescence emission spectra of purified FAD-RNA (CoES7) and free FAD are shown in Figure 4A. Except for a lower fluorescence intensity of FAD-RNA relative to that of free FAD, both spectra are similar, with no apparent spectral shift. At the same concentration, the fluorescence intensity of FAD-RNA is about $35-40 \%$ of that of free FAD in $20 \mathrm{mM}$ MES, pH 6.0. This decrease in fluorescence of FAD upon covalent coupling to RNA is presumably due to quenching by FAD-linked RNA, because similar concentrations of RNA added to free FAD solutions $(0.1 \mu \mathrm{M})$ do not affect the fluorescence emission of FAD (results not shown).

To demonstrate the effect of covalent linkage to RNA, emission spectra of FAD-RNA were taken before and after complete digestion of FAD-RNA by nuclease P1 (Fig. 4B). Although no spectral shift was observed, fluorescence intensity increased 2.5-fold, to the level of free FAD. This result confirms that covalent linkage to RNA considerably reduces the fluorescence quantum yield of FAD.

The effect of covalent coupling to RNA can be further demonstrated through FAD-RNA denaturation by urea. As shown in Figure 4C, high concentrations of urea significantly increase the fluorescence intensity of FAD-RNA. In 4 and $7 \mathrm{M}$ urea (containing $0.5 \mathrm{M} \mathrm{NaAc}, \mathrm{pH}$ 5.2), the fluorescence intensity of FAD-RNA was enhanced 2.5- and 5.1-fold, respectively. On the other hand, the fluorescence of free FAD increased only 1.7- and 2.7-fold under the same conditions (emission spectra not shown).

Combining the results of Figure 4, it becomes clear that the reduced fluorescence of FAD-RNA relative to that of free FAD is not a result of the covalent linkage itself between FAD and the $5^{\prime}$ end of RNA. The fluorescence decrease is rather caused by a folded RNA conformation. In free FAD, stacking between the isoalloxazine and the adenine significantly quenches the fluorescence intensity of isoalloxazine $(14,15)$. In the presence of $7 \mathrm{M}$ urea, unstacking of the isoalloxazine and the adenine of free FAD results in a fluorescence enhancement of 2.7-fold. Under the same conditions,

Figure 4. Fluorescence properties of CoES7 FAD-RNA. (A) Fluorescence emission spectra of free FAD and CoES7 FAD-RNA. The spectra were recorded from the same concentration $(0.1 \mu \mathrm{M})$ of FAD and FAD-RNA in $20 \mathrm{mM}$ MES buffer, pH 6.0, at an excitation wavelength of $370 \mathrm{~nm}$. While there is no apparent spectral shift between FAD and FAD-RNA, coupling FAD to the $5^{\prime}$ end of RNA results in a 2.6-fold decrease in fluorescence intensity. (B) Fluorescence intensity comparison of FAD-RNA before and after nuclease P1 digestion. FAD-RNA $(0.1 \mu \mathrm{M})$ was dissolved in $10 \mathrm{mM}$ $\mathrm{NaAc}\left(\mathrm{pH} 5.2\right.$ ) containing $0.4 \mathrm{mM} \mathrm{ZnCl}_{2}$. After its emission spectrum was recorded, $1 \mu \mathrm{l}$ of nuclease $\mathrm{P} 1(1 \mathrm{U} / \mu \mathrm{l})$ was added to a total volume of $120 \mu \mathrm{l}$ of FAD-RNA solution. Nuclease P1 digestion of FAD-RNA causes a significant increase in fluorescence intensity (2.5-fold increase). (C) Fluorescence enhancement of FAD-RNA in the presence of high concentrations of urea. Three separate FAD-RNA $(0.1 \mu \mathrm{M})$ solutions were prepared in $50 \mathrm{mM} \mathrm{NaAc}(\mathrm{pH} 5.2), 4 \mathrm{M}$ urea and $7 \mathrm{M}$ urea (containing $50 \mathrm{mM} \mathrm{NaAc}$ ). From $50 \mathrm{mM} \mathrm{NaAc}$ to $4 \mathrm{M}$ and $7 \mathrm{M}$ urea, FAD-RNA fluorescence intensity increases 2.5 - and 5.1-fold, respectively.
FAD-RNA is denatured (both RNA conformation and the stacking of the isoalloxazine and the adenine of the FAD moiety), leading to a 5.1-fold increase in fluorescence intensity. The larger increase in fluorescence intensity of FAD-RNA over free FAD in the presence of $7 \mathrm{M}$ urea reflects the contribution of the folded RNA conformation, which is corroborated by the 2.5-fold fluorescence reduction from free FAD to FAD-RNA (Fig. 4A). Both FAD and FAD-RNA exhibit similar fluorescence quantum yields (the same fluorescence intensity from the same concentrations of FAD and
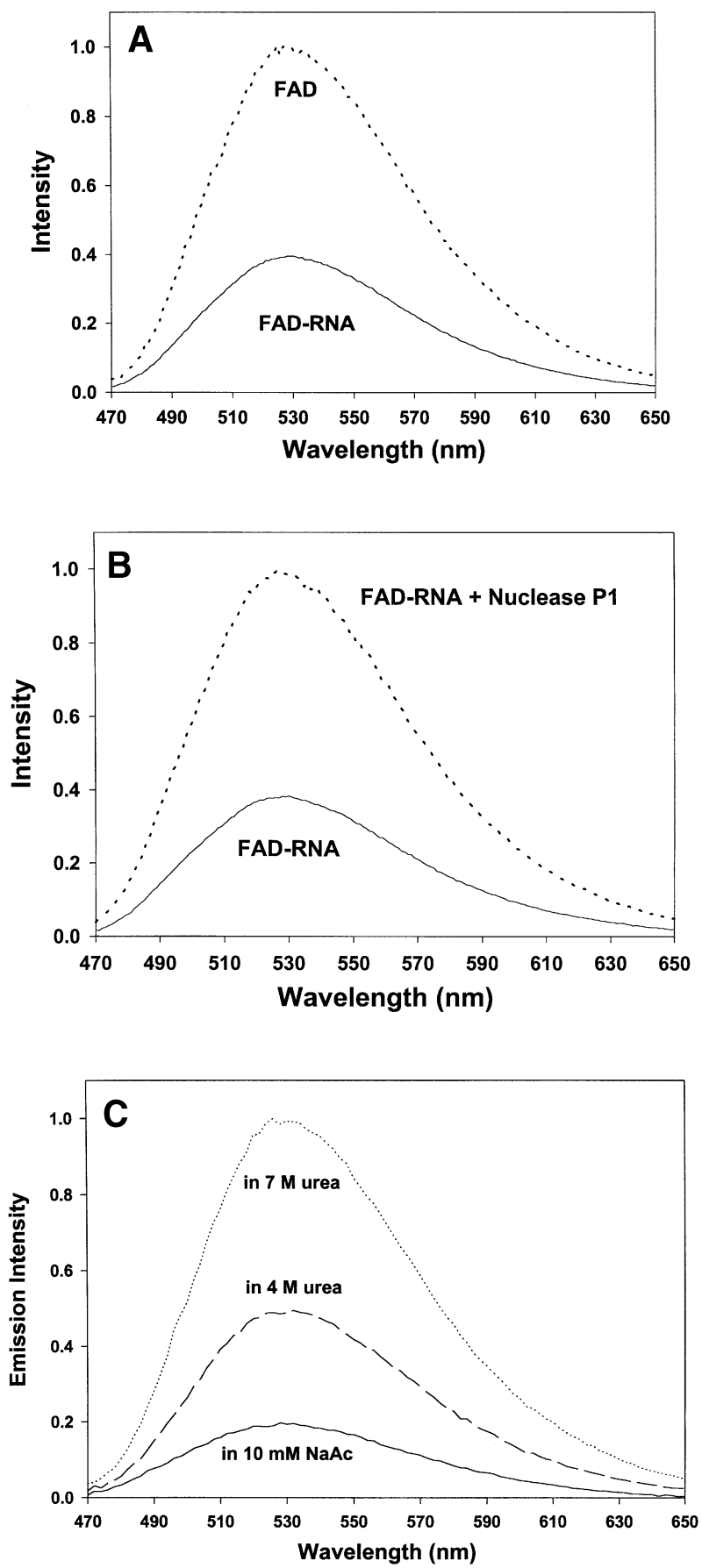
FAD-RNA) in $7 \mathrm{M}$ urea, indicating that the isoalloxazine group in FAD and FAD-RNA is in a similar chemical environment under these conditions. Thus, introducing the covalent bond by itself between FAD and RNA does not appear to affect FAD fluorescence. However, the covalent linkage is necessary to bridge the interaction between FAD and RNA, because similar RNA concentrations added to free FAD solutions do not affect FAD fluorescence intensity.

\section{DISCUSSION}

Using a transcription promoter sequence derived from $\mathrm{T} 7$ class II promoters (12), we have demonstrated that transcription can be initiated by adenosine derivatives, including ATP (4), De-P-CoA, NAD and FAD (Fig. 2). Adenosine can be used to prepare adenosine-initiated RNA with free $5^{\prime}$ hydroxyl groups, permitting easy ${ }^{32} \mathrm{P}$-labeling of the $5^{\prime}$ end of RNA by polynucleotide kinase (unpublished results). Because only the adenosine group is required for recognition by T7 RNA polymerase to initiate transcription, a large number of adenosine derivatives can be used to prepare adenosine derivative-linked RNA. In addition to the three coenzymes used in this study (CoA, NAD and FAD), a variety of biologically active molecules can be linked to the $5^{\prime}$ end of RNA. These include the coenzymes $S$-adenosylcysteine, $S$-adenosylhomocysteine (AdoHcy) and $S$-adenosylmethionine (SAM), the sugar-containing molecule adenosine $5^{\prime}$ diphosphoglucose (ADPG) and the signaling molecules diadenosine polyphosphates $\mathrm{Ap}(3) \mathrm{A}$ and $\mathrm{Ap}(4) \mathrm{A}$ (16-22). Chemically modified adenosine derivatives such as fluorescent ATP derivatives (23-25) may also be used to prepare 5' fluorophore-linked RNA. A similar strategy has been used to prepare $5^{\prime}$ fluorophore-linked RNA from guanosine derivatives (26). While other RNA polymerases such as Escherichia coli RNA polymerase can be used to prepare adenosine derivative-initiated RNA (27), the well-defined T3, T7 and SP6 promoters, high yields of transcription and easy preparation of these phage RNA polymerases make them the method of choice for in vitro RNA preparation $(28,29)$.

Convenient and efficient in vitro transcription methods have played an important role in the advancement of RNA research by providing easily available RNA with defined sequences. However, all the current methods require a guanosine derivative as the transcription initiator under the T3, T7 and SP6 promoters $(28,29)$. Although other nucleotides such as adenosine and cytidine derivatives have been shown to be able to initiate transcription with certain sequences $(30,31)$, the initiation sites are not well defined. The yields are low (30), and they usually result from non-templated incorporation or omission of one or two nucleotides (31). Our previous report (4) and this study establish that ATP and adenosine derivatives can initiate transcription of any defined sequences with high efficiency, thereby providing researchers with choices of different $5^{\prime}$ ends of RNA. The yield of adenosine derivativeinitiated RNA seems to be slightly lower (70-80\%) than guanosine derivative-initiated RNA from one study (4). However, during our in vitro selection experiments, we consistently obtained high yields of adenosine derivativeinitiated random RNA libraries, reaching as high as $100 \mu \mathrm{g}$ RNA/100 $\mu \mathrm{l}$ transcription under standard transcription conditions. Therefore, 300 copies of 50 nt RNA molecules can be synthesized from a single DNA template.

In transcription where an adenosine derivative (R-A) other than ATP is present, both normal RNA (pppRNA) and adenosine derivative-linked RNA (R-ARNA) are produced. Although high concentrations of adenosine derivatives (such as De-P-CoA, NAD and FAD) lead to high relative yields of R-ARNA over total RNA (pppRNA and R-ARNA), total RNA yields may decrease at high concentrations of adenosine derivatives. To balance the total RNA yields and relative yields of R-ARNA, transcription in the presence of $1 \mathrm{mM}$ of each of the four nucleoside triphosphates and $4 \mathrm{mM}$ of an adenosine derivative is highly recommended. However, if higher relative yields of R-ARNA are desired and lower total RNA yields are acceptable, higher concentrations of adenosine derivatives or lower concentrations of ATP may be used. At $>8 \mathrm{mM}$ adenosine derivative concentrations, total RNA yields can be significantly lower. At $0.2-0.5 \mathrm{mM}$ ATP and 4 $\mathrm{mM}$ De-P-CoA, transcription produces $>50 \%$ CoA-RNA with an $\sim 20-50 \%$ reduction in total RNA yields. Optimization of transcription conditions such as magnesium concentration may improve the total RNA yield and relative yield of R-ARNA.

Among the four normal ribonucleosides and ribonucleotides (A, G, C and $\mathrm{U}$ ), adenosine and its derivatives are overwhelmingly represented and play dominant roles in the living cell (32). The functions of a large number of protein enzymes in metabolism depend on the three common adenosine-containing coenzymes, CoA, NAD and FAD. The unique structures and universal utilization of these coenzymes in all living organisms suggest their ancient history, plausibly as far back as in a pre-protein world as first hypothesized by White $(2,3)$. Our previous demonstration of RNA-catalyzed CoA, NAD and FAD synthesis from their corresponding precursors (4) supports the existence and functioning of CoA, NAD and FAD in the RNA world (5). Further supporting evidence comes from the finding of plausible availability of coenzyme precursors through prebiotic synthesis (33-36). To demonstrate the utilization of coenzymes by RNA, we have successfully isolated ribozymes capable of CoA thioester synthesis from random CoA-linked RNA libraries (prepared from De-P-CoA-initiated transcription) (7). An independent selection has also yielded acyl-CoA-synthesizing ribozymes (6). Although the utilization of NAD and FAD by ribozymes has yet to be shown, our adenosine derivative-initiated transcription method should provide easy preparation of NAD- and FAD-linked random RNA libraries, thereby facilitating isolation via SELEX of RNA sequences capable of catalyzing NAD and FAD redox chemistry (i.e. electron/ hydride transfer reactions).

The fluorescence property of FAD-RNA may be used to investigate the RNA structure and function relationship. The defined $5^{\prime}$ end location of the fluorophore FAD and easy preparation of FAD-RNA by in vitro transcription should be advantageous for structural investigation of RNA by steadystate fluorescence spectroscopy. The lifetime of the fluorophore FAD in FAD-RNA may change and correlate with its chemical environment, thereby providing a sensor for RNA folding and conformation through time-resolved fluorescence spectroscopy $(37,38)$. Furthermore, another fluorophore (either a donor or an acceptor) can be introduced at other 
locations in the RNA to allow investigation of spatial arrangement of the RNA around its $5^{\prime}$ end by fluorescence resonance energy transfer (FRET) (39-41). For small RNA fragments, fluorophores can be added at specific locations by chemical synthesis $(42,43)$. However, introduction of a specific chemical group (including a fluorophore) at a defined location in relatively large RNA may involve multiple steps of transcription, chemical synthesis and ligation $(44,45)$. With an appropriate fluorescence donor or acceptor, FAD-initiated transcription can significantly reduce the number of steps needed to prepare RNA suitable for a broad range of FRET studies.

FAD-RNA may find other applications such as fluorescence detection of complementary RNA/DNA sequences. Different $5^{\prime}$ end FAD-labeled RNA sequences can be conveniently synthesized by this in vitro transcription method, in theory, to allow detection of a large number of different RNA/DNA sequences.

In addition, the reduced form of NAD (i.e. NADH) is also highly fluorescent (46). Therefore, NADH-RNA may find a wide range of applications, as discussed above. NADH and FAD have different spectral properties and lifetimes (46) and may respond differently to their RNA environment. FAD-RNA and NADH-RNA may accordingly be combined in RNA structural investigations by fluorescence spectroscopy.

\section{ACKNOWLEDGEMENTS}

I would like to thank Tricia Coleman for her assistance with PAGE analysis, Peter Butko for discussions and the use of the fluorometer and the members of our laboratories for comments on the manuscript. This work was supported by a NSF grant MCB9974487 and a NASA grant NAG5-10668.

\section{REFERENCES}

1. Benner,S.A., Ellington,A.D. and Tauer,A. (1989) Modern metabolism as a palimpsest of the RNA world. Proc. Natl Acad. Sci. USA, 86, 7054-7058.

2. White,H.B. (1976) Coenzymes as fossils of an earlier metabolic state. J. Mol. Evol., 7, 101-104.

3. White,H.B. (1982) Evolution of coenzymes and the origin of pyridine nucleotides. In Everse,J., Anderson,B. and Yu,K.-S. (eds), The Pyridine Nucleotide Coenzymes. Academic Press, New York, NY, pp. 1-17.

4. Huang,F., Bugg,C.W. and Yarus,M. (2000) RNA-catalyzed CoA, NAD and FAD synthesis from phosphopantetheine, NMN and FMN. Biochemistry, 39, 15548-15555.

5. Gilbert,W. (1986) The RNA world. Nature (Lond.), 319, 618.

6. Jadhav,V.R. and Yarus,M. (2002) Acyl-CoAs from coenzyme ribozymes. Biochemistry, 41, 723-729.

7. Coleman,T.M. and Huang,F. (2002) RNA-catalyzed thioester synthesis. Chem. Biol., 9, 1227-1236.

8. Ellington,A.D. and Szostak,J.W. (1990) In vitro selection of RNA molecules that bind specific ligands. Nature, 346, 818-822.

9. Tuerk,C. and Gold,L. (1990) Systematic evolution of ligands by exponential enrichment: RNA ligands to bacteriophage T4 DNA polymerase. Science, 249, 505-510.

10. Robertson,D.L. and Joyce,G.F. (1990) Selection in vitro of an RNA enzyme that specifically cleaves single-stranded DNA. Nature, 344, 467-468.

11. Jaschke,A. (2001) Artificial ribozymes and deoxyribozymes. Curr. Opin. Struct. Biol., 11, 321-326.

12. Dunn,J.J. and Studier,F.W. (1983) Complete nucleotide sequence of bacteriophage T7 DNA and the locations of T7 genetic elements. J. Mol. Biol., 166, 477-535.
13. Santoro,S.W. and Joyce,G.F. (1997) A general purpose RNA-cleaving DNA enzyme. Proc. Natl Acad. Sci. USA, 94, 4262-4266.

14. Bessey,O.A., Lowry,O.H. and Love,R.H. (1949) The fluorometric measurement of the nucleotides of riboflavin and their concentration in tissues. J. Biol. Chem., 180, 755-769.

15. Weber,G. (1950) Fluorescence of riboflavin and flavin-adenine dinucleotide. Biochem. J., 47, 114-121.

16. Rapaport,E. and Zamecnik,P.C. (1976) Presence of diadenosine $5^{\prime}, 5^{\prime \prime \prime}$ P1, P4-tetraphosphate (Ap4A) in mamalian cells in levels varying widely with proliferative activity of the tissue: a possible positive "pleiotypic activator". Proc. Natl Acad. Sci. USA, 73, 3984-3988.

17. Varshavsky,A. (1983) Diadenosine 5', 5'"-P1, P4-tetraphosphate: a pleiotropically acting alarmone? Cell, 34, 711-712.

18. Bochner,B.R., Lee,P.C., Wilson,S.W., Cutler,C.W. and Ames,B.N. (1984) AppppA and related adenylylated nucleotides are synthesized as a consequence of oxidation stress. Cell, 37, 225-232.

19. Johnstone,D.B. and Farr,S.B. (1991) AppppA binds to several proteins in Escherichia coli, including the heat shock and oxidative stress proteins DnaK, GroEL, E89, C45 and C40. EMBO J., 10, 3897-3904.

20. Schluter,H., Offers,E., Bruggemann,G., van der Giet,M., Tepel,M., Nordhoff,E., Karas,M., Spieker,C., Witzel,H. and Zidek,W. (1994) Diadenosine phosphates and the physiological control of blood pressure. Nature, 367, 186-188.

21. Baxi,M.D. and Vishwanatha,J.K. (1995) Diadenosine polyphosphates: their biological and pharmacological significance. J. Pharmacol. Toxicol. Methods, 33, 121-128.

22. Kisselev,L.L., Justesen,J., Wolfson,A.D. and Frolova,L.Y. (1998) Diadenosine oligophosphates (Ap(n)A), a novel class of signaling molecules? FEBS Lett., 427, 157-163.

23. Yarbrough,L.R. (1978) Synthesis and properties of a new fluorescent analog of ATP: adenosine-5'-triphosphoro-gamma-1-(5-sulfonic acid) napthylamidate. Biochem. Biophys. Res. Commun., 81, 35-41.

24. Yarbrough,L.R., Schlageck,J.G. and Baughman,M. (1979) Synthesis and properties of fluorescent nucleotide substrates for DNA-dependent RNA polymerases. J. Biol. Chem., 254, 12069-12073.

25. Wu,F.Y., Abdulwajid,A.W. and Solaiman,D. (1986) Synthesis and properties of adenosine-5' -triphosphoro-gamma-1-(5-sulfonic acid)naphthyl ethylamidate: a fluorescent nucleotide substrate for DNAdependent RNA polymerase from Escherichia coli. Arch. Biochem. Biophys., 246, 564-571.

26. Sastry,S. (2001) A fluorescence-based assay for transcription using a novel fluorescent GTP analogue. Biophys. Chem., 91, 191-208.

27. Yarbrough,L.R., Schlageck,J.G. and Baughman,M. (1979) Synthesis and properties of fluorescent nucleotide substrates for DNA-dependent RNA polymerases. J. Biol. Chem., 254, 12069-12073.

28. Milligan,J.F., Groebe,D.R., Witherell,G.W. and Uhlenbeck,O.C. (1987) Oligoribonucleotide synthesis using T7 RNA polymerase and synthetic DNA templates. Nucleic Acids Res., 15, 8783-8798.

29. Pokrovskaya,I.D. and Gurevich,V.V. (1994) In vitro transcription: preparative RNA yields in analytical scale reactions. Anal. Biochem., 220, 420-423.

30. Nam,S.C. and Kang,C.W. (1988) Transcription initiation site selection and abortive initiation cycling of phage SP6 RNA polymerase. J. Biol. Chem., 263, 18123-18127.

31. Helm,M., Brule,H., Giege,R. and Florentz,C. (1999) More mistakes by T7 RNA polymerase at the $5^{\prime}$ ends of in vitro-transcribed RNAs. RNA, $\mathbf{5}$, 618-621.

32. Wilson,J.E. (1984) Some thoughts on the evolutionary basis for the prominent role of ATP and ADP in cellular energy metabolism. J. Theor. Biol., 111, 615-623.

33. Dowler,M.J., Fuller,W.D., Orgel,L.E. and Sanchez,R.A. (1970) Prebiotic synthesis of propiolaldehyde and nicotinamide. Science, 169, 1320-1321.

34. Miller,S.L. and Schlesinger,G. (1993) Prebiotic syntheses of vitamin coenzymes: I. cysteamine and 2-mercaptoethanesulfonic acid (coenzyme M). J. Mol. Evol., 36, 302-307.

35. Miller,S.L. and Schlesinger,G. (1993) Prebiotic syntheses of vitamin coenzymes: II. Pantoic acid, pantethenic acid and composition of coenzyme A. J. Mol. Evol., 36, 308-314.

36. Keefe,A.D., Newton,G.L. and Miller,S.L. (1995) A possible prebiotic synthesis of pantetheine, a precursor to coenzyme A. Nature, 373, 683-685.

37. Visser,A.J.W.J. and Muller,F. (1980) Time-resolved fluorescence on flavins and flavoproteins. Methods Enzymol., 66, 373-385. 
38. Klostermeier,D. and Millar,D.P. (2001) Time-resolved fluorescence resonance energy transfer: a versatile tool for the analysis of nucleic acids. Biopolymers, 61, 159-179.

39. Tuschl,T., Gohlke,C., Jovin,T.M., Westhof,E. and Eckstein,F. (1994) A three-dimensional model for the hammerhead ribozyme based on fluorescence measurements. Science, 266, 785-789.

40. Clegg,R.M. (1995) Fluorescence resonance energy transfer. Curr. Opin. Biotechnol., 6, 103-110.

41. Klostermeier,D. and Millar,D.P. (2001) RNA conformation and folding studied with fluorescence resonance energy transfer. Methods, 23, 240-254.
42. Earnshaw,J. and Gait,M.J. (1998) Modified oligoribonucleotides as sitespecific probes of RNA structure and function. Biopolymers, 48, 39-55.

43. Scaringe,S.A., Wincott,F.E. and Caruthers,M.H. (1998) Novel RNA synthesis method using $5^{\prime}$-O-silyl-2'-O-orthoester protecting groups. J. Am. Chem. Soc., 120, 11820-11821.

44. Moore,M.J. and Query,C.C. (2000) Joining of RNAs by splinted ligation. Methods Enzymol., 317, 109-123.

45. Golden,B.L. (2000) Heavy atom derivatives of RNA. Methods Enzymol., 317, 124-132.

46. Lakowicz,J.R. (1999) Principles of Fluorescence Spectroscopy. Plenum Publishers, New York, NY. 